\title{
Quercetin promotes the osteogenic differentiation of rat mesenchymal stem cells via mitogen-activated protein kinase signaling
}

\author{
YANG LI $^{1 *}$, JIEFANG WANG $^{1 *}$, GUANGMING CHEN $^{1}$, SHUIWANG FENG $^{1}$, \\ PANPAN WANG $^{2}$, XIAOFENG ZHU ${ }^{2}$ and RONGHUA ZHANG ${ }^{1}$
}

${ }^{1}$ Department of Traditional Chinese Medicine, College of Pharmacy; ${ }^{2}$ Department of Traditional Chinese Medicine,
First Affiliated Hospital of Jinan University, Jinan University, Guangzhou, Guangdong 510632, P.R. China

Received December 2, 2014; Accepted March 5, 2015

DOI: $10.3892 / \mathrm{etm} .2015 .2388$

\begin{abstract}
The aim of the present study was to investigate the effects of quercetin on the mitogen-activated protein kinase (MAPK) signaling pathway in the osteogenic differentiation of rat mesenchymal stem cells (MSCs). A 3-(4,5-dimethylthiazol-2-yl)-2,5-diphenyltetrazolium bromide assay and an alkaline phosphatase (ALP) assay were used to determine the effects of quercetin (concentrations of $0.1,1$ and $10 \mu \mathrm{mol} / \mathrm{l}$ ) on the proliferation and differentiation of MSCs and the expression of ALP, respectively. In addition, through the introduction of inhibitors of p38 MAPK, extracellular signal-regulated kinase (ERK)1/2 and c-Jun $\mathrm{NH}_{2}$-terminal kinase (JNK), the effects of quercetin on the proteins, ALP, collagen type I (COL I) and bone $\gamma$-carboxyglutamate protein (BGP), which are indicators of osteogenic differentiation, were investigated. Immunoblotting was performed to determine the phosphorylation levels of p38 MAPK, ERK1/2 and JNK, while fluorescent quantitative polymerase chain reaction was used to determine the mRNA expression levels of transforming growth factor (TGF)- $\beta 1$, bone morphogenetic protein (BMP)-2 and core binding factor $(\mathrm{CBF}) \alpha 1$. At all the concentrations tested, the concentrations of 10,1 and $0.1 \mu \mathrm{mol} / 1$ quercetin were shown to promote the differentiation of MSCs and the expression of ALP, in which the concentration of $10 \mu \mathrm{mol} / \mathrm{l}$ was optimal. When compared with the control group, the phosphorylation levels of $\mathrm{p} 38$ MAPK, ERK1/2 and JNK, the protein expression levels of ALP, COL I and BGP, and the mNRA expression
\end{abstract}

Correspondence to: Professor Ronghua Zhang, Department of Traditional Chinese Medicine, College of Pharmacy, Jinan University, 601 West Huangpu Avenue, Guangzhou, Guangdong 510632, P.R. China

E-mail: tzrh@jnu.edu.cn

*Contributed equally

Key words: bone-marrow mesenchymal stem cells, osteogenic differentiation, mitogen-activated protein kinase, mitogen-activated protein kinase signaling pathway levels of TGF- $\beta 1$, BMP- 2 and Cbf $\alpha 1$ were increased in the quercetin-treated group. However, with the introduction of inhibitors, the levels of phosphorylated p38 MAPK, ERK1/2 and JNK, and the protein expression levels of ALP, COL I and BGP decreased. Furthermore, the mRNA expression levels of TGF- $\beta 1$, BMP- 2 and CBF $\alpha 1$ decreased in the quercetin + SP600125 (inhibitor of JNK) and quercetin + PD98059 (inhibitor of ERK1/2) groups. Therefore, quercetin was demonstrated to promote the osteogenic differentiation of MSCs by activating the MAPK signaling pathway. The ERK1/2 and JNK signaling pathways regulate the expression of TGF- $\beta 1$, BMP-2 and CBF 1 . Thus, activation of the ERK1/2 and JNK signaling pathways may play a leading role in the quercetin-promoted osteogenic proliferation and differentiation of MSCs.

\section{Introduction}

Mesenchymal stem cells (MSCs) are a type of adult stem cell that are capable of multipotential differentiation. The cells can be easily separated, extracted, cultured and amplified. Through migration, proliferation and differentiation, MSCs form osteoblasts (OBs) in the absorption areas of bones, and subsequently mediate the formation of new bone tissue to maintain the dynamic balance in bone function (1). The differentiation of MSCs to OBs is a complex process that is regulated and controlled by multiple signaling pathways, and with which the mitogen-activated protein kinase (MAPK) signaling pathway is closely associated. In addition, previous studies have indicated that the p38 MAPK, extracellular signal-regulated kinase (ERK)1/2 and c-Jun $\mathrm{NH}_{2}$-terminal kinase (JNK) signaling pathways play important roles in inducing the differentiation of MSCs to OBs (2-4).

Quercetin is one of the most ubiquitous bioflavonoids, occurring widely in plants in the form of glycosides or aglycone, which exert a number of pharmacological effects, functioning as antioxidants (5), anti-inflammatory agents (6), antitumor agents (7) and metabolic regulators (8). Quercetin has also been shown to exert a positive pharmacological effect on bone metabolism (9-11), although the underlying mechanism of action is yet to be fully elucidated. However, there have been a limited number of studies investigating the effects of 
quercetin on MSCs. In the present study, MSCs from Sprague Dawley rats were treated with quercetin, with or without the presence of inhibitors against p38 MAPK, ERK1/2 and JNK, in order to investigate the roles of the p38 MAPK, ERK1/2 and JNK signaling pathways in quercetin-induced osteogenic differentiation. Thus, the cellular and molecular biological mechanisms of quercetin in protecting the bone mass were clarified, and the cellular and molecular basis underlying the activity of quercetin in preventing and treating bone metabolic diseases, including osteoporosis, were defined.

\section{Materials and methods}

Animals and drugs. In total, five female specific-pathogen free Sprague Dawley rats (age, 3 months; weight, 230 $\pm 10 \mathrm{~g}$ ), provided by the Laboratory Animal Center of Southern Medical University (approval no. Guangdong SCXK 2006-0015; Guangzhou, China), were used for the extraction of MSCs. The present study was approved by the Animal Ethics Committee of Jinan University (Guangzhou, China).

Quercetin was provided by the National Institute for the Control of Pharmaceutical and Biological Products (Beijing, China; lot no. 10081-2009072).

Reagents. Superior fetal bovine serum (FBS) was purchased from Tianjin Haoyang Biological Manufacture Co., Ltd (Wuhan, China). Minimum essential medium $\alpha(\mathrm{MEM} \alpha)$ was obtained from HyClone (GE Healthcare, Logan, UT, USA), and an alkaline phosphatase (ALP) assay kit was purchased from Nanjing Jiancheng Bioengineering Institute (Nanjing, China). SB203580 (SB; inhibitor of p38 MAPK), PD98059 (PD; inhibitor of ERK1/2) and SP600125 (SP; inhibitor of JNK) were obtained from Millipore Corporation (Bedford, MA, USA). Trypsin was purchased from Amresco LLC (Solon, $\mathrm{OH}$, USA). Dimethyl sulfoxide (DMSO) was from obtained from Sigma-Aldrich (St. Louis, MO, USA) and TRIzol reagent was purchased from Invitrogen Life Technologies (Carlsbad, CA, USA). Furthermore, 3-(4,5-dimethylthiazol-2-yl)-2,5-diphenyltetrazolium bromide (MTT) and a bicinchoninic acid (BCA) Protein Assay kit were purchased from the Beyotime Institute of Biotechnology (Haimen, China). A rabbit anti-rat glyceraldehyde phosphate dehydrogenase polyclonal antibody, rabbit anti-rat ERK1/2 and rabbit anti-rat phosphorylated ERK1/2 (p-ERK1/2) primary antibodies and a goat anti-rabbit secondary antibody were obtained from Cell Signaling Technology, Inc. (Danvers, MA, USA). The SYBR ${ }^{\circledR}$ Green polymerase chain reaction (PCR) Master Mix was purchased from Toyobo Co., Ltd. (Osaka, Japan), and rat bone $\gamma$-carboxyglutamate protein (BGP) and rat collagen type I (COL I) ELISA kits were purchased from Hebei Bacarray Biotechnology Development Co., Ltd (Xingtai, China). An enhanced chemiluminescence (ECL) western blotting detection system was purchased from Pierce Biotechnology, Inc. (Rockford, IL, USA). All other reagents and chemicals were of analytical grade and were available commercially.

Extraction and culture of MSCs. Anesthesia was induced by an intramuscular injection of $20 \%$ urethane $(6 \mathrm{mg} / \mathrm{kg}$; Aladdin Chemical, Shanghai, China). The Sprague Dawley rats were sacrificed by cervical dislocation, and steeped in $75 \%$ ethanol for $10 \mathrm{~min}$. The tibias and femurs were separated, and the soft tissues were removed. The bones were further soaked in $75 \%$ ethanol for 10-30 sec on the benchtop. After the tibias and femurs were washed five times with D-Hanks solution, the metaphyses on both sides of the tibias and femurs were resected and the marrow cavity was washed repeatedly with MEM/ $\alpha$ containing $10 \%$ FBS using a 5-ml syringe. The cells were repeatedly pipetted with a 1-ml syringe to form a single-cell suspension at a density of $1.0 \times 10^{9}$ cells $/ 1$. A $25-\mathrm{cm}^{2}$ culture flask was seeded with the single-cell suspension and placed in an incubator at $37^{\circ} \mathrm{C}$ under $5 \% \mathrm{CO}_{2}$. After $24 \mathrm{~h}$, half the medium was replaced, and after $48 \mathrm{~h}$, all the medium was replaced. The culture medium was changed every three days. The third-generation cells were used for the subsequent experiments.

MSC subculture and purification. After culture for 7-10 days, the first-generation cells reached $90 \%$ confluence and the culture medium was discarded. The cells were washed three times with D-Hanks solution and digested with $0.25 \%$ trypsin for 1-2 min. Under an inverted optical microscope (Olympus IX51; Olympus Optical Co. Ltd., Tokyo, Japan), the adherent cells appeared contracted, almost circular and with broad intercellular spaces. Fresh complete culture medium was subsequently added (with a straw to avoid bubbles) to terminate the digestion. The single-cell suspension was diluted $1: 2$ with the culture medium, and seeded into two $25-\mathrm{cm}^{2}$ culture flasks. The culture flasks were placed in an incubator at a constant temperature of $37^{\circ} \mathrm{C}$, with $5 \% \mathrm{CO}_{2}$ and saturated humidity. The medium was replaced every three days. After 7-9 days, when the cells had reached 70 or $80 \%$ confluence, the culture process was repeated. This natural purification method was used to purify the MSCs.

Identification of MSCs. The morphology of the MSCs cultured for 24 h, 3 days, 7 days or 12 days was observed with an inverted optical microscope.

\section{Cell induction}

Cells of the third generation, at a density of $1.0 \times 10^{5}$ cells $/ \mathrm{ml}$ per well, were seeded in six-well flat-bottomed plates $(60 \mathrm{~mm})$ with a coverslip in each well to prepare the cell slides. The slides were used for electron microscopic analysis to determine the phenotype of the MSCs.

Osteogenic differentiation. When the cells reached $80 \%$ confluence, the MSCs were cultured in human mesenchymal stem cell osteogenic differentiation medium (Cyagen Biosciences Inc., Santa Clara, CA, USA), which was replaced every three days. The cells were induced with osteogenic medium and cultured for 21 days. After induction for 12 days, the cell slides containing the OBs were washed 3-5 times with D-Hanks solution and fixed in acetone for 10 min. Subsequently, the slides were washed 3-5 times with distilled water, incubated with $\beta$-glycerophosphate at $37^{\circ} \mathrm{C}$ for $6 \mathrm{~h}$, and washed several times with distilled water. The slides were placed into $2 \%$ nitric acid cobalt for 3-5 min, rinsed several times with distilled water and soaked in $1 \%$ ammonium sulfide for $2 \mathrm{~min}$. Finally, the slides were rinsed with distilled water and dried naturally. Following osteogenic induction for 21 days, the cell slides were washed 
3-5 times with D-Hanks solution, fixed with 75\% alcohol for 15 min, washed 3-5 times with D-Hanks solution again, and stained with $0.2 \%$ Alizarin red (Sigma-Aldrich) for $30 \mathrm{~min}$ to observe the mineralization nodes at a magnification of $\mathrm{x} 3$.

Adipogenic differentiation. The MSCs were subsequently cultured in adipogenic differentiation medium (Cyagen Biosciences Inc.) which was replaced every 3 days and cultured for 15 days. After 15 days, the cell slides containing adipocytes were observed and photographed microscopically. In addition, the cells were stained with Oil Red O (Amresco LLC) to quantify the extent of adipogenic differentiation. The cells were washed several times with distilled water prior to photographing.

Detection of surface markers. The third-generation cells were digested with $0.25 \%$ trypsin, and complete medium was added to terminate the digestion reaction. The cell suspension was centrifuged at $800 \mathrm{x}$ g to collect the cells. The cells were subsequently resuspended in D-Hanks solution and centrifuged three times $800 \mathrm{x}$ g. D-Hanks solution was then added to resuspend the cells as a single-cell suspension at a density of $1 \times 10^{6}$ cell $/ \mathrm{ml}$. An aliquot $(500 \mathrm{ml})$ of the single-cell suspension was placed in each of four 1.5- $\mu 1$ Eppendorf tubes, and incubated with pure culture medium and monoclonal antibodies (fluorescein-isothiocyanate-conjugated mouse anti-rat CD29, CD34 or CD45; Cell Signaling Technology, Inc., Beverly, $\mathrm{MA}, \mathrm{USA}$ ) at $37^{\circ} \mathrm{C}$ for $30 \mathrm{~min}$. After the cells were washed three times with D-Hanks solution, the cells were quantified by flow cytometry on ice using an EPICS-XL flow cytometer (Beckman Coulter, Brea, CA, USA).

MTT assay. Third-generation cells, digested with $0.25 \%$ trypsin, were resuspended and seeded in 96-well plates at a density of $5.0 \times 10^{6}$ cells $/ 1$ per well. Following incubation at $37^{\circ} \mathrm{C}$ under $5 \% \mathrm{CO}_{2}$, the MSCs were divided into a control group and groups treated with different concentrations of quercetin $(0.01,0.1,1,10$ or $100 \mu \mathrm{mol} / 1)$. Six replicate samples were included in each group. The cells were incubated with $\mathrm{MEM} / \alpha$ containing $10 \%$ FBS for $24 \mathrm{~h}$. The various concentrations of quercetin were added to the respective groups, and the cells were cultured with the same concentration of MEM/ $\alpha$ for an additional $24 \mathrm{~h}$. After the culture medium was discarded, the cells were cultured with $20 \mu \mathrm{l}$ MTT solution $(5 \mathrm{mg} / \mathrm{ml})$ in an incubator at $37^{\circ} \mathrm{C}$ under $5 \% \mathrm{CO}_{2}$ for $4 \mathrm{~h}$. The MTT solution was subsequently discarded and $100 \mu \mathrm{l}$ DMSO was added to each well. The culture plate was oscillated (E24 incubator shaker; Eppendorf AG, Hamburg, Germany) at a low speed until the crystals were completely dissolved. The absorbance value of each sample was measured with a microplate reader (MK3; Thermo, Minnesota, MN, USA) at a wavelength of $490 \mathrm{~nm}$.

ALP activity assay. MSCs were seeded in 96-well plates at a density of $1.0 \times 10^{8}$ cells $/ 1$ and cultured in an incubator at $37^{\circ} \mathrm{C}$ under conditions of $5 \% \mathrm{CO}_{2}$ until they reached $80 \%$ confluence. After $24 \mathrm{~h}$, the MSCs were divided into a control group and different concentration quercetin treatment groups $(0.01$, $0.1,1,10$ or $100 \mu \mathrm{mol} / \mathrm{l})$, with six replicates in each group. The cells were incubated with MEM/ $\alpha$ containing $10 \%$ FBS for $24 \mathrm{~h}$. Quercetin was subsequently added to the respective groups, and the cells were cultured with the same volume of MEM/ $\alpha$ for $72 \mathrm{~h}$. The culture medium in each well was discarded and the cells were washed two or three times with D-Hanks solution. A 1-ml sample of cell lysate was added to each well and incubated at $4^{\circ} \mathrm{C}$ for $30 \mathrm{~min}$, after which the cell lysate was collected. The total concentration of protein was determined using the BCA Protein Assay kit, while ALP activity was measured using the ALP kit.

Groups and treatment methods. Cells were seeded in six $60-\mathrm{mm}$ flat-bottomed plates at a density of $1.0 \times 10^{8}$ cells $/ 1$, and divided into a control group, quercetin group, quercetin + PD group, quercetin + SB group, quercetin + SP group and quercetin $+\mathrm{PD}+\mathrm{SB}+\mathrm{SP}$ group. The cells were incubated with serum-free MEM/ $\alpha$ for $24 \mathrm{~h}$, and the inhibitor-treated groups were incubated with the respective inhibitors for $30 \mathrm{~min}$. To inhibit the p38 MAPK, JNK or ERK1/2 signaling pathways, the cells were incubated with a p38 MAPK inhibitor (SB), JNK inhibitor (SP) or ERK1/2 inhibitor (PD) for $30 \mathrm{~min}$. Quercetin was then added to each well. The final concentrations were as follows: PD ERK1/2 pathway inhibitor, $10 \mu \mathrm{mol} / 1$; SB p38 MAPK pathway inhibitor, $3 \mu \mathrm{mol} / \mathrm{l}$; and SP JNK pathway inhibitor, $5 \mu \mathrm{mol} / 1$. The final concentration of quercetin was $10 \mu \mathrm{mol} / \mathrm{l}$.

ELISAs. After the cells were incubated with serum free $\mathrm{MEM} / \alpha$ for $24 \mathrm{~h}$, the control group was incubated with the $\mathrm{MEM} / \alpha$ medium and the inhibitor-treated groups were incubated with the respective inhibitors for $72 \mathrm{~h}$. Quercetin was then added to the inhibitor-treated wells and the final concentrations were as previously described; the culture medium was discarded. Subsequently, the cells were washed three times with D-Hanks solution, and a $150-\mu 1$ sample of cell lysate was added to each well and incubated at $4^{\circ} \mathrm{C}$ for $30 \mathrm{~min}$, after which the cell lysate was collected. The BCA Protein Assay kit was used to determine the total concentration of protein, and the ALP assay kit was used to quantify the expression of ALP. In addition, ELISAs were performed to quantify the protein expression of COL I and BGP.

Immunoblotting analysis. Inhibitors were added to the cell suspensions as aforementioned. Following culture of the cells for $24 \mathrm{~h}$, the culture medium was discarded and the cells were washed three times with D-Hanks solution. An aliquot (100 $\mu \mathrm{l})$ of cell lysate was then added to each well and the plates were incubated at $4^{\circ} \mathrm{C}$ for $30 \mathrm{~min}$, after which the cell lysate was collected. The BCA Protein Assay kit was used to determine the total protein concentration. The cell proteins $(30 \mu \mathrm{g})$ were separated by sodium dodecyl sulfate- $12 \%$ polyacrylamide gel electrophoresis and transferred onto polyvinylidene difluoride membranes, which were blocked with buffer containing $0.05 \%$ Tween-20 and 5\% defatted milk. Subsequently, the membranes were reacted with the primary antibodies at $4{ }^{\circ} \mathrm{C}$ for $12 \mathrm{~h}$, washed three times with D-Hanks solution and reacted with the secondary antibodies at room temperature for $1 \mathrm{~h}$. Next, the membranes were washed and rinsed with ECL detection reagents, and the banding patterns images were captured with ECL software (ChemiDoc ${ }^{\mathrm{TM}} \mathrm{XRS}+$ system; Bio-Rad, Hercules, CA, USA). The primary antibodies (all from Cell Signaling Technology, Inc.) included rabbit anti-rat ERK1/2 (\#4695), 
Table I. Primer sequences used for fluorescent quantitative PCR.

\begin{tabular}{ll}
\hline Gene name & \multicolumn{1}{c}{ Primer sequence } \\
\hline TGF- $\beta 1$ & F: 5'-TGCTTCAGCTCCACAGAGAA-3' \\
& R: 5'-TGGTTGTAGAGGGCAAGGAC-3' \\
BMP-2 & F: 5'-GTGAGGATTAGCAGGTCTTTG-3' \\
& R: 5'-CACCCCACATCACTGAAGTC-3' \\
CBF $\alpha 1$ & F: 5'-GATGCCTTAGTGCCCAAATGT-3' \\
& R: 5'-GGCTGAAGGGTGAAGAAAGC-3' \\
18S rRNA & F: 5'-CCTGGATACCGCAGCTAGGA-3' \\
& R: 5'-GCGGCGCAATACGAATGCCCC-3'
\end{tabular}

PCR, polymerase chain reaction; TGF, transforming growth factor; $\mathrm{BMP}$, bone morphogenetic protein; $\mathrm{CBF}$, core binding factor; $\mathrm{F}$, forward; R, reverse; rRNA, ribosomal RNA.

rabbit anti-rat JNK (\#9258), rabbit anti-rat p38 MAPK (\#8690), rabbit anti-rat P-ERK1/2 (\#4094), rabbit anti-rat P-p38 MAPK (\#9215), rabbit anti-rat P-JNK (\#4668) and rabbit anti-rat glyceraldehyde 3-phosphate dehydrogenase (GAPDH; \#5174); all at a dilution of 1:1,000. The goat anti-rabbit secondary antibody (\#7074; Cell Signaling Technology, Inc.) was applied to rabbit $\mathrm{IgG}-\mathrm{H} \& \mathrm{~L}$ (horseradish peroxidase) at a dilution of 1:3,000. The immunoreactive bands were visualized with enhanced chemiluminescent substrates on an X-ray film (Eastman Kodak, Rochester, NY, USA), and the expression levels of the proteins were detected with the system described in the Quantity One 1-D ${ }^{\circledR}$ analysis software manual (Bio-Rad Laboratories, Inc. Hercules, CA, USA).

Fluorescent quantitative PCR. MSCs were cultured for $24 \mathrm{~h}$ with the appropriate inhibitors, as previously described. The total RNA was extracted from the cells using TRIzol reagent and the total RNA ( $1 \mu \mathrm{g})$ was reverse transcribed by conversion to cDNA using the iScript cDNA Synthesis kit according to the manufacturer's instructions (Bio-Rad). The cDNA was used as the template for fluorescent quantitative PCR to detect the mRNA expression levels of transforming growth factor (TGF)- $\beta 1$, bone morphogenetic protein (BMP)-2 and core binding factor $(\mathrm{CBF}) \alpha 1$. The details of the primers are provided in Table I. Quantitative PCR was performed in 96-well plates with a reaction volume of $20 \mu \mathrm{l}$ per well, which included $10 \mu \mathrm{l}$ 2X SYBR ${ }^{\circledR}$ Green PCR Master Mix, $5 \mu \mathrm{l} \mathrm{cDNA,} 0.5 \mu \mathrm{l}$ each

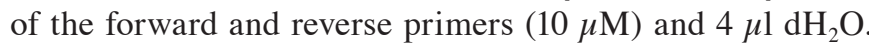
An ABI PRISM ${ }^{\circledR} 7500$ Sequence Detection System (Applied Biosystems Life Technologies, Foster City, CA, USA) was used for the quantitative analysis under the following conditions: Initial denaturation at $95^{\circ} \mathrm{C}$ for $5 \mathrm{~min}$, followed by 40 cycles of denaturation at $95^{\circ} \mathrm{C}$ for $15 \mathrm{sec}$, annealing at $60^{\circ} \mathrm{C}$ for $15 \mathrm{sec}$ and elongation at $72^{\circ} \mathrm{C}$ for $32 \mathrm{sec}$. The fluorescent signal was collected at the end of the second step during each cycle and plotted on a graph against the reaction cycle index. Each sample was analyzed in triplicate and the average cycle threshold $(\mathrm{Ct})$ was used to analyze the mRNA expression levels of each gene using the ${ }^{\Delta \Delta} \mathrm{Ct}$ quantification method.
Statistical analysis. SPSS 16.0 software (SPSS, Inc., Chicago, IL, USA) was used to perform all statistical calculations. Data are expressed as the mean \pm standard deviation. One-way analysis of variance was used to compare the differences between the experimental groups, where $\mathrm{P}<0.05$ was considered to indicate a statistically significant difference.

\section{Results}

Cell morphology. Morphological assessment revealed that the MSCs adhered $24 \mathrm{~h}$ after extraction. The non-adherent hematopoietic cells were removed when the culture medium was totally replaced. The MSCs began to adapt to the in vitro culture and had an almost circular morphology, as shown in Fig. 1A. After 3 days, prominent filopodia extensions, short rod or triangular cells, cellular protrusions and an oblate nuclear morphology were observed, indicating that the cells had divided rapidly, as shown in Fig. 1B. The MSCs were stretched, and formed large clusters of stellate cells. On day 7 , the majority of the cells had gradually became fusiform, with cell colonies beginning to form, and the cells undergoing rapid proliferation. These cells were used for culture, as shown in Fig. 1C. On day 12, the second-generation MSCs had reached $90 \%$ confluence. The cells grew in a swirl shape and established a stable-fibroblast-like phenotype, as shown in Fig. 1D. On day 15, scanning electron microscopy was used to observe the MSCs. The cells appeared as long fusiform shapes or polygons, with a lot of intracellular granular material and slender microspines and silk on their surfaces, surrounded by a number of matrix components, as shown in Fig. 1E.

Effects of osteogenic or adipogenic induction. When the MSCs were cultured in osteogenic medium, their morphology gradually changed from long fusiform cells to rectangular or polygonal cells. In addition, the quantity of the extracellular matrix increased in the clusters of cells. Numerous black granules were observed in the extracellular and cellular matrix, whereas the color of the nuclei became lighter. Following culture for 12 days, the cells stained positively for ALP and a number of brown or black granular precipitates appeared in the cytoplasm, as shown in Fig. 2A. The cells were stained with Alizarin red to detect the mineralization. On day 21, Alizarin red staining revealed that a number of cells had gathered into nodules, in which the cells took on a cubic or cone shape. Furthermore, the cells were aligned in a multilayer structure and the secretion of a large numbers of granules was evident. Almost the whole cell layer was heavily covered with a mineralized matrix, as shown in Fig. 2B. When the MSCs were cultured in adipogenic medium, the morphology was shown to gradually become round or oval. On day 15, adipogenic differentiation was confirmed through staining with Oil Red O, as shown in Fig. 2C. In addition, the adipocytes were readily identified by their intracellular accumulation of neutral lipids, as shown in Fig. 2D.

Detection of cell surface markers. Detection of the surface makers, CD29, CD34 and CD45, in the third-generation MSCs was performed using flow cytometry (Fig. 3). The majority (99.5\%) of the MSCs expressed the mesenchymal surface 
A

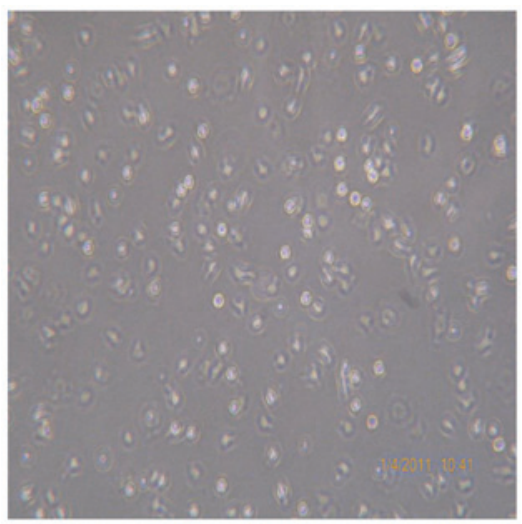

C

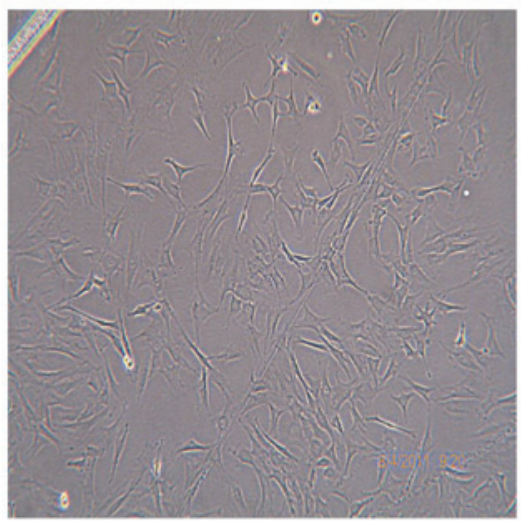

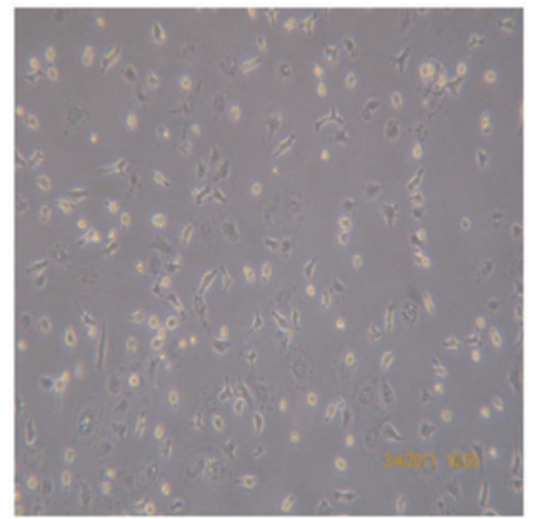

D

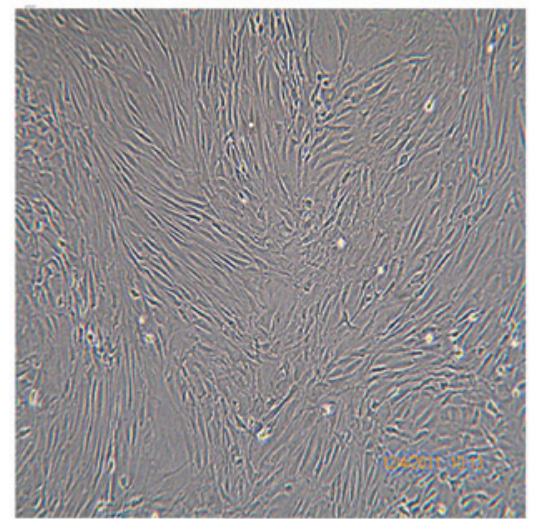

$\mathbf{E}$

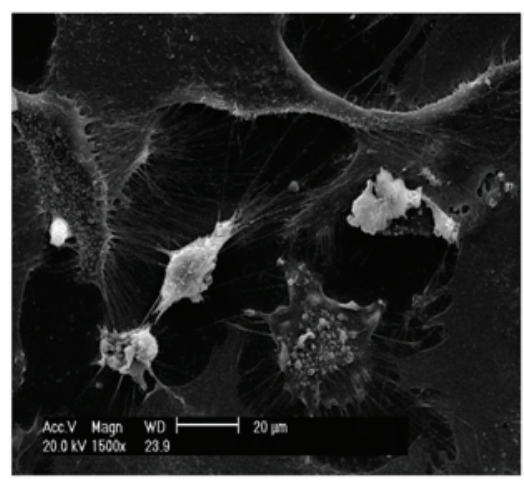

Figure 1. Morphological features of the primary cells cultured for (A) $24 \mathrm{~h}$, (B) 3 days, (C) 7 days and (D) 12 days (magnification, x100). (E) Morphology of the third-generation mesenchymal stem cells (magnification, $\mathrm{x} 1,500)$, as observed with scanning electron microscopy.

marker, CD29, whereas only $3.3 \%$ of the cells expressed the hematopoietic marker, CD34, and only $0.3 \%$ of the MSCs expressed the hematopoietic marker, CD45.

Effects of different quercetin concentrations on MSC proliferation and ALP expression. In the MTT assay, the absorbance values of the groups treated with $0.1,1$ or $10 \mu \mathrm{mol} / 1$ quercetin were significantly higher compared with those of the control group $(\mathrm{P}<0.05)$; there were no statistically significant differences between the three groups themselves. The absorbance values of the groups treated with 0.01 or $100 \mu \mathrm{mol} / 1$ quercetin were not significantly different compared with the control group $(\mathrm{P}>0.05)$. In addition, the expression of ALP was significantly higher in the cells treated with $0.1,1$ and $10 \mu \mathrm{mol} / \mathrm{l}$ quercetin, as compared with the control group $(\mathrm{P}<0.05)$. Furthermore, ALP expression was significantly higher following treatment with $10 \mu \mathrm{mol} / \mathrm{l}$ quercetin when compared with the levels following treatment with 0.1 or $1 \mu \mathrm{mol} / 1$ quercetin $(\mathrm{P}<0.05)$. Therefore, $10 \mu \mathrm{mol} / 1$ quercetin was the optimal concentration for promoting the osteogenic differentiation of MSCs, as shown in Table II.

\section{ALP activity, COL I content and BGP content in the different} groups. Protein expression levels of ALP, COL I and BGP increased significantly in the quercetin group when compared with that in the control group $(\mathrm{P}<0.05)$. However, with the introduction of inhibitors, the expression of ALP, COL I and BGP decreased in the quercetin $+\mathrm{PD}$, quercetin $+\mathrm{SP}$ and quercetin $+\mathrm{PD}+\mathrm{SB}+\mathrm{SP}$ groups, as compared with that in the quercetin group $(\mathrm{P}<0.05)$. In the quercetin $+\mathrm{SB}$ group, the expression of ALP was lower than that in the control and quercetin groups $(\mathrm{P}<0.05)$; however, the expression of COL I was higher compared with the control group $(\mathrm{P}<0.05)$, although the value did not differ significantly from the quercetin group 

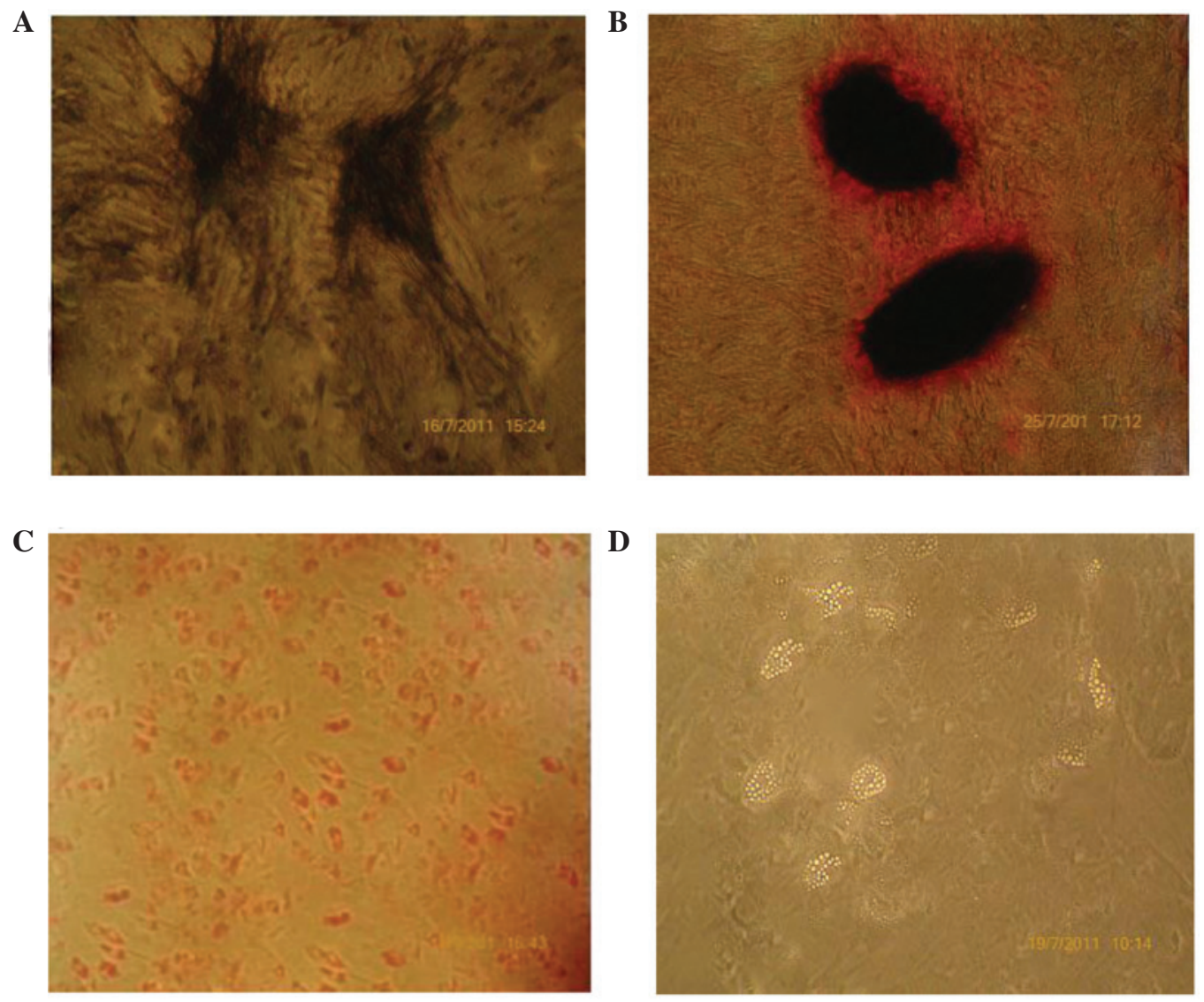

Figure 2. Morphological features of mesenchymal stem cells (MSCs) undergoing differentiation following induction with osteogenic or adipogenic medium. Following the addition of osteogenic medium, the long spindle-shaped cells gradually became rectangular or polygonal, and the amount of extracellular matrix increased in the cell clusters. A number of black granules became darker and the color of the nuclei became lighter. (A) At $12 \mathrm{~h}$ after induction, the cells stained positively for alkaline phosphatase and a number of brown or black granular precipitates were visible in the cytoplasm (magnification, x200). (B) Alizarin red staining was used to detect mineralization. On day 21, Alizarin red staining revealed that almost the whole cell layer was heavily covered with a mineralized matrix (magnification, x200). (C) On day 15, adipogenic differentiation was confirmed when the cells were stained with Oil Red O (magnification, x100), (D) Adipocytes were readily identified morphologically by their intracellular accumulation of neutral lipids (magnification, x100).

$(\mathrm{P}>0.05)$. Furthermore, the expression of BGP was lower than that in the quercetin group $(\mathrm{P}<0.05)$; however, the difference was not statistically significant when compared with the control group ( $\mathrm{P}>0.05)$, as shown in Table III.

Phosphorylation of JNK, ERK1/2 and p38 MAPK in the different groups. As shown in Fig. 4, the phosphorylation of p38 MAPK (P-p38MAPK), ERK1/2 (P-ERK1/2) and JNK $(\mathrm{P}-\mathrm{JNK})$ increased significantly $(\mathrm{P}<0.05)$ in the quercetin group when compared with the control group. However, the phosphorylation of p38 MAPK, ERK1/2 and JNK decreased $(\mathrm{P}<0.05)$ in the quercetin $+\mathrm{SB}$, quercetin $+\mathrm{PD}$, quercetin $+\mathrm{SP}$ and quercetin $+\mathrm{PD}+\mathrm{SB}+\mathrm{SP}$ groups when compared with the quercetin group, as shown in Fig. 4.

mRNA expression levels of TGF- $\beta 1, B M P-2$ and $C B F \alpha 1$ in the different groups. The mRNA expression levels of TGF- $\beta 1$, BMP-2 and $\mathrm{CBF} \alpha 1$ increased in the quercetin group when compared with the control group $(\mathrm{P}<0.05)$. However, the mRNA expression levels of TGF- $\beta 1$, BMP- 2 and CBF $\alpha 1$ decreased significantly $(P<0.05)$ in the quercetin $+P D$ and quercetin + SP groups when compared with those in the quercetin group. In the quercetin $+\mathrm{SB}$ group, the expression of BMP-2 decreased $(\mathrm{P}<0.05)$; however, there was no statisti- cally significant difference in the expression levels of TGF- $\beta 1$ or $\mathrm{CBF} \alpha 1(\mathrm{P}>0.05)$ when the compared with the quercetin group. Furthermore, in the quercetin + PD + SB + SP group, the mRNA expression level of $\mathrm{CBF} \alpha 1$ decreased $(\mathrm{P}<0.05)$; however, there were no statistically significant differences in the expression levels of TGF- $\beta 1$ and BMP- 2 when compared with the quercetin group, as shown in Fig. 5.

\section{Discussion}

Reduced proliferation and osteogenesis of MSCs, and the increased adipogenesis of MSCs, are known to serve important roles in the pathogenesis of osteoporosis (12). In the present study, MSCs were shown to differentiate into OBs and adipocytes in osteogenic and adipogenic induction medium, respectively. In addition, third-generation cells expressed the mesenchymal surface marker, CD29, whereas the cells seldom expressed the hematopoietic markers, CD34 and CD45. These results indicated that the cells extracted were MSCs. The osteogenesis of MSCs is affected by several factors, including hormones and cytokines, and the process is regulated by a number of signaling pathways. The MAPK signaling pathway is known to be closely associated with the osteogenesis of MSCs $(13,14)$. 
A

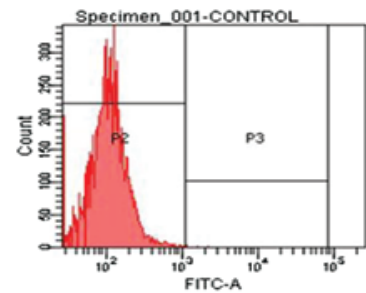

Tube: CONTROL
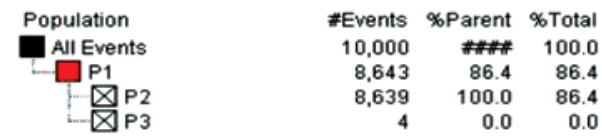

B
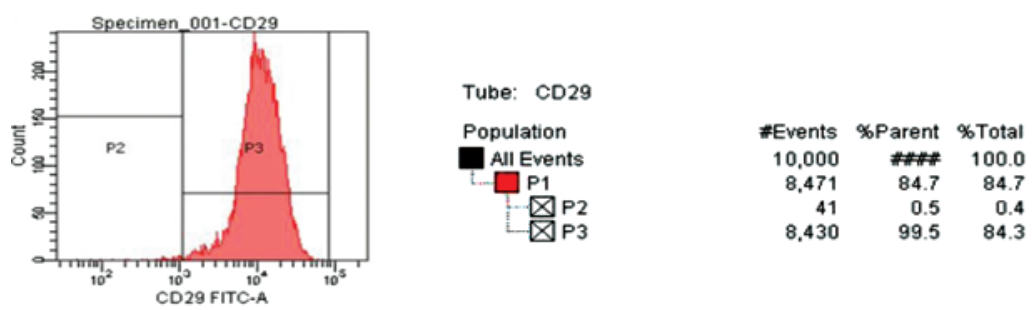

C
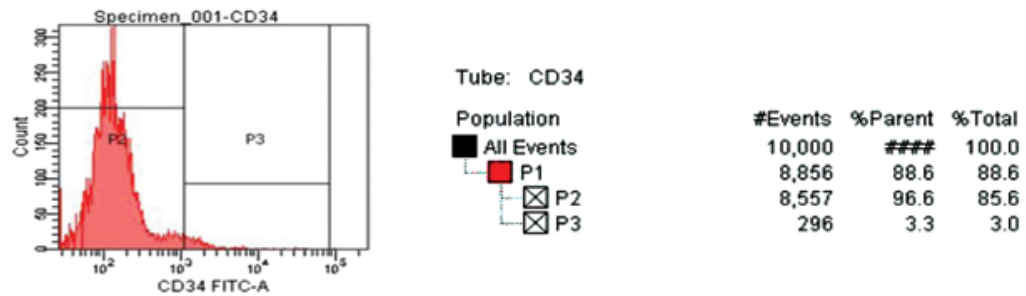

D
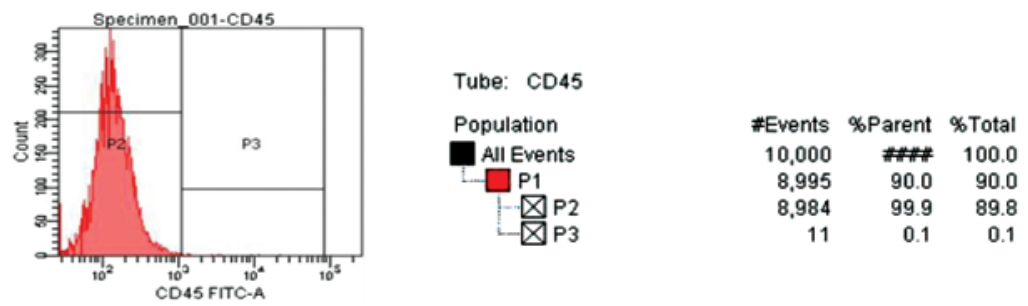

Figure 3. Detection of mesenchymal stem cell surface markers using flow cytometry. Third-generation cells were shown to express the mesenchymal surface marker, CD29; however, negligible expression of hematopoietic markers was observed. (A) Control; (B) CD29 (99.5\%); (C) CD34 (3.3\%); and (D) CD45 (0.3\%). FITC, fluorescein-isothiocyanate.

Table II. Effects of different concentrations of quercetin on MSC proliferation and ALP expression.

\begin{tabular}{lcc}
\hline Quercetin $(\mu \mathrm{mol} / \mathrm{l})$ & A-value & ALP $(\mathrm{U} / \mathrm{g}$ protein $)$ \\
\hline 0 & $0.809 \pm 0.029$ & $13.176 \pm 0.449^{\mathrm{b}}$ \\
0.01 & $0.779 \pm 0.064$ & $14.285 \pm 0.392^{\mathrm{b}}$ \\
0.1 & $0.946 \pm 0.121^{\mathrm{a}}$ & $18.267 \pm 0.265^{\mathrm{a}, \mathrm{b}}$ \\
1 & $0.976 \pm 0.121^{\mathrm{a}}$ & $20.128 \pm 0.131^{\mathrm{a}, \mathrm{b}}$ \\
10 & $0.955 \pm 0.046^{\mathrm{a}}$ & $26.204 \pm 0.848^{\mathrm{a}}$ \\
100 & $0.763 \pm 0.090$ & $12.501 \pm 0.250^{\mathrm{a}, \mathrm{b}}$
\end{tabular}

Results are expressed as the mean \pm standard deviation $(\mathrm{n}=6) .{ }^{\mathrm{a}} \mathrm{P}<0.05$, vs. control group; ${ }^{\mathrm{b}} \mathrm{P}<0.05$, vs. $10 \mu \mathrm{mol} / \mathrm{l}$ quercetin group. From the 3-(4,5-dimethylthiazol-2-yl)-2,5-diphenyltetrazolium bromide assay, the A-values for the groups treated with 10,1 or $0.1 \mu \mathrm{mol} / 1$ quercetin were significantly higher when compared with the control group $(\mathrm{P}<0.05)$, although they did not differ significantly from one another $(\mathrm{P}>0.05)$. ALP expression was significantly higher in the groups treated with $0.1,1$ and $10 \mu \mathrm{mol} / \mathrm{l}$ quercetin when compared with the control group $(\mathrm{P}<0.05)$, and ALP expression was significantly higher in the $10 \mu \mathrm{mol} / 1$ group when compared with the 0.1 and $1 \mu \mathrm{mol} / 1$ groups $(\mathrm{P}<0.05)$. Therefore, $10 \mu \mathrm{mol} / 1$ quercetin was the optimal concentration for promoting the osteogenic differentiation of MSCs. MSC, mesenchymal stem cell; ALP, alkaline phosphatase; A, absorbance.
The MAPK signaling pathway is one of the most important signal transduction systems in vivo. Subgroups of the MAPK signaling pathway primarily include ERK1/2, JNK, p38 MAPK and ERK5, which are involved in the regulation of cell growth, proliferation, differentiation, survival and apoptosis, in response to a variety of extracellular stimuli $(15,16)$. The ERK1/2, JNK and p38 MAPK signaling pathways have been previously reported to be essential for the osteoblastic differentiation of MSCs (17-19).

MSCs express a variety of bone marker proteins in different stages of their osteogenic differentiation. In the early stage, the expression of ALP and COL I is predominant, followed by the expression of matrix proteins. Thereafter, BGP is expressed and the extracellular matrix is calcified. The cooperation of the matrix proteins ensures the maturation of the extracellular matrix and initiates OB-forming bone tissues (17). A variety of growth factors are involved in the process of maturation and bone mineralization, which are very important for proper bone remodeling and repair. Of these, TGF- $\beta$ is the most important. Members of the TGF- $\beta$ family include the various TGF- $\beta$ isoforms, activins, BMPs and other associated factors (18). In general, TGF- $\beta 1$ not only enhances preosteoblast proliferation and extracellular matrix synthesis, but also counters the effect of BMP-2 on OB differentiation (19). Furthermore, TGF- $\beta 1$ and 
Table III. ALP activity, COL I content and BGP content in the different groups.

\begin{tabular}{lccc}
\hline Group & ALP (U/g protein) & COL I $\left(10^{3}\right.$ U/g protein $)$ & BGP $(\mu \mathrm{g} / \mathrm{g}$ protein $)$ \\
\hline Control & $20.363 \pm 1.182$ & $3.007 \pm 0.102$ & $5.842 \pm 0.234$ \\
QUE & $35.499 \pm 1.208^{\mathrm{b}}$ & $3.756 \pm 0.163^{\mathrm{b}}$ & $7.580 \pm 0.245^{\mathrm{a}}$ \\
QUE + PD & $23.880 \pm 1.035^{\mathrm{d}}$ & $3.160 \pm 0.076^{\mathrm{d}}$ & $6.437 \pm 0.273^{\mathrm{c}}$ \\
QUE + SB & $10.590 \pm 1.033^{\mathrm{d}, \mathrm{b}}$ & $3.822 \pm 0.031^{\mathrm{b}}$ & $5.600 \pm 0.226^{\mathrm{c}}$ \\
QUE + SP & $29.670 \pm 1.032^{\mathrm{d}}$ & $3.002 \pm 0.020^{\mathrm{d}}$ & $4.143 \pm 0.025^{\mathrm{c}}$ \\
QUE + PD + SB + SP & $19.837 \pm 1.001^{\mathrm{d}}$ & $2.790 \pm 0.045^{\mathrm{d}}$ & $4.249 \pm 0.111^{\mathrm{c}}$ \\
\hline
\end{tabular}

Results are expressed as the mean \pm standard deviation $(\mathrm{n}=3)$. ${ }^{\mathrm{a}} \mathrm{P}<0.05$ and ${ }^{\mathrm{b}} \mathrm{P}<0.01$, vs. control group; ${ }^{\mathrm{c}} \mathrm{P}<0.05$ and ${ }^{\mathrm{d}} \mathrm{P}<0.01$, vs. $\mathrm{QUE}$ group. Expression levels of ALP, COL I and BGP increased significantly in the quercetin group when compared with the control group (P<0.05). Following treatment with the corresponding inhibitors, the expression levels of ALP, COL I and BGP decreased in the quercetin + PD, quercetin $+\mathrm{SP}$ and quercetin $+\mathrm{PD}+\mathrm{SB}+\mathrm{SP}$ groups when compared with the quercetin group $(\mathrm{P}<0.05)$. In the quercetin $+\mathrm{SB}$ group, the expression of ALP was lower than that in the control or quercetin groups $(\mathrm{P}<0.05)$; COL I expression was higher than that in the control group $(\mathrm{P}<0.05)$, however, did not differ significantly from the quercetin group $(\mathrm{P}>0.05)$; BGP expression was lower than that in the quercetin group $(\mathrm{P}<0.05)$, but did not differ significantly from the control group (P>0.05). QUE, quercetin; ALP, alkaline phosphatase; COL I, collagen type I; BGP, bone $\gamma$-carboxyglutamate protein; PD, PD98059; SB, SB203580; SP, SP600125.

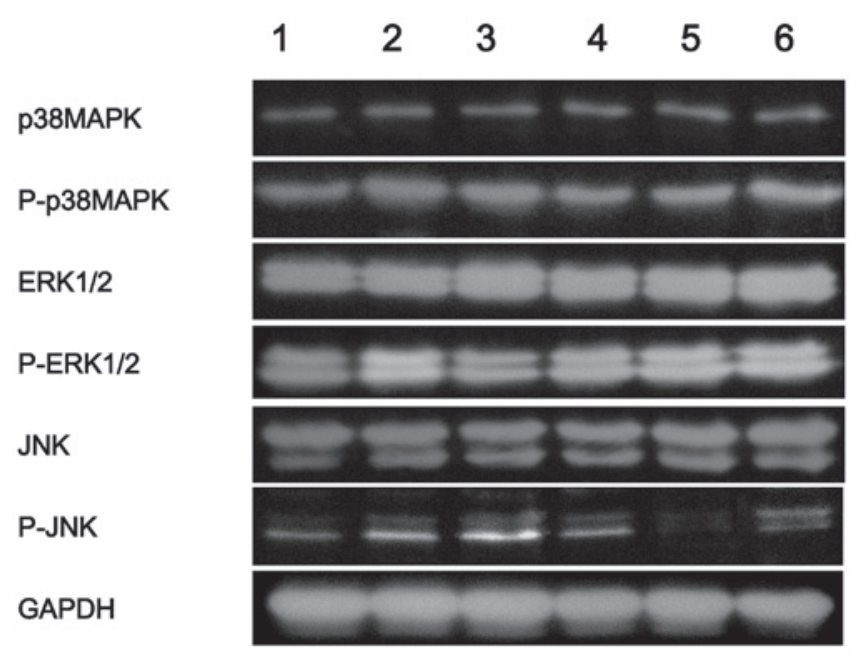

Figure 4. Western blot analysis showing the protein expression of P-p38 MAPK, P-ERK1/2 and P-JNK in each group. Lane 1, control; lane 2, quercetin group; lane 3: quercetin + PD group; lane 4, quercetin + SB group; lane 5, quercetin + SP group; lane 6, quercetin + PD + SB + SP group. PD, PD98059; SB, SB203580; SP, SP600125; MAPK, mitogen-activated protein kinase; ERK, extracellular signal-regulated kinase; JNK, c-Jun $\mathrm{NH}_{2}$-terminal kinase; GAPDH, glyceraldehyde phosphate dehydrogenase; P, phosphorylated.

BMP-2 promote the osteoblastic and chondrogenic differentiation of MSCs and induce ectopic bone formation. In addition, TGF- $\beta 1$ has been reported to induce the expression of specific genes, such as BGP, COL I and ALP (20). Therefore, TGF- $\beta 1$ and BMP-2 play important roles in the osteoblastic differentiation of MSCs $(21,22)$. CBF $\alpha 1$, an important signaling molecule in the process of bone formation (23), is a specific transcription factor belonging to the runt-domain gene family (24). The molecule is essential for the maturation of OBs and the osteogenesis of MSCs. In addition, $\mathrm{CBF} \alpha 1$ participates in the regulation of genes and matrix gene expression in OBs. The major regulatory genes involved in the development of bone formation include ALP, COL I and BGP (25). Therefore, the present study investigated the protein expression levels of ALP, COL I and BGP to analyze osteoblastic differentiation, and the

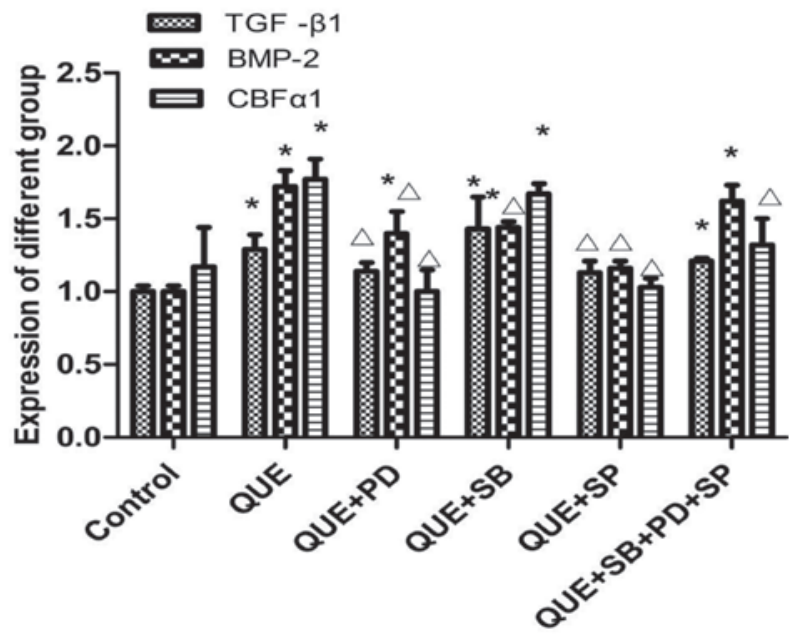

Figure 5. mRNA expression levels of TGF- $\beta 1$, BMP-2 and CBF $\alpha 1$ in each group. Results are expressed as the mean \pm standard deviation $(n=3)$. ${ }^{*} \mathrm{P}<0.05$, vs. control group; ${ }^{\Delta} \mathrm{P}<0.05$, vs. QUE group. QUE, quercetin; TGF, transforming growth factor; $\mathrm{BMP}$, bone morphogenetic protein; $\mathrm{CBF}$, core binding factor; PD, PD98059; SB, SB203580; SP, SP600125.

mRNA expression levels of TGF- $\beta 1$, BMP-2 and CBF $\alpha 1$ to investigate the mechanism underlying the effect of quercetin on the proliferation and differentiation of MSCs.

In the present study, the results demonstrated that the activity of ALP and the protein levels of BGP and the structural protein, COL I, were significantly increased by quercetin. The optimal concentration of quercetin for promoting the osteogenic differentiation of rat MSCs was determined to be $10 \mu \mathrm{mol} / \mathrm{l}$. Quercetin was also demonstrated to stimulate the p38 MAPK, ERK1/2 and JNK signaling pathways. Conversely, the expression levels of ALP, COL I and BGP were reduced following inhibition of the JNK, ERK1/2 or all three signaling pathways. However, when an inhibitor of the p38 MAPK signaling pathway was introduced, the expression levels of ALP and BGP decreased, while the expression of COL I was not affected. Therefore, quercetin promotes the expression of ALP, COL I and BGP, and the ERK1/2, p38 MAPK and JNK 
signaling pathways are essential for the quercetin-induced osteoblastic differentiation of MSCs.

In the present study, quercetin was shown to promote the expression of TGF- $\beta 1$, BMP- 2 and CBF $\alpha 1$. The expression of BMP-2 was reduced when the p38 MAPK signaling pathway was inhibited, whereas there was no statistically significant difference in the expression of TGF- $\beta 1$ or $\mathrm{CBF} \alpha 1$. These results demonstrate that p38 MAPK regulates the expression of BMP-2; however, this signaling pathway has no significant effect on the expression of TGF- $\beta 1$ or CBF $\alpha 1$. Thus, quercetin stimulates the osteogenic differentiation of MSCs, which is mediated by the activation of the p38 MAPK signaling pathway. The mRNA expression levels of TGF- $\beta 1, \mathrm{BMP}-2$ and $\mathrm{CBF} \alpha 1$ were reduced following the addition of an inhibitor of the JNK or ERK1/2 signaling pathway. However, when inhibitors of the JNK, ERK1/2 and p38 MAPK signaling pathways were introduced, the expression of $\mathrm{CBF} \alpha 1$ decreased, while the expression levels of TGF- $\beta 1$ and BMP-2 were unaffected. Therefore, quercetin increases the expression of TGF- $\beta 1, \mathrm{BMP}-2$ and $\mathrm{CBF} \alpha 1$, and subsequently promotes osteoblastic differentiation, which is primarily mediated by the ERK $1 / 2$ and JNK signaling pathways.

In conclusion, the ability of quercetin to induce the osteogenic differentiation of MSCs can be weakened by blocking the p38 MAPK, ERK1/2 or JNK signaling pathway. Therefore, the ERK1/2 and JNK signaling pathways may play leading roles in the regulation of quercetin-induced osteogenic differentiation of rat MSCs.

\section{Acknowledgements}

This study was supported by grants from the National Natural Science Foundation of China (nos. 81173619 and 81473509), the Cultivation and Innovation Fund for Scientific Research of Jinan University Youth Fund Project (no. 21612341), Guangdong Provincial Natural Science Foundation (no. S2012040007531) and the Fundamental Research Funds for the Central Universities (no. 21614309).

\section{References}

1. Hadjidakis DJ and Androulakis II: Bone remodeling. Ann NY Acad Sci 1092: 385-396, 2006

2. Fu L, Tang T, Miao Y, Zhang S, Qu Z and Dai K: Stimulation of osteogenic differentiation and inhibition of adipogenic differentiation in bone marrow stromal cells by alendronate via ERK and JNK activation. Bone 43: 40-47, 2008.

3. Luo Y, Liu Y and Zhang KQ: The effect of MAPK signal pathway in the process of osteogenic differentiation in mesenchymal stem cells. Jilin Yi Xue 29: 443-445, 2008 (In Chinese).

4. Wang Y, Li J, Wang Y, Lei L, Jiang C, An S, Zhan Y, Cheng Q, Zhao Z, Wang J and Jiang L: Effects of hypoxia on osteogenic differentiation of rat bone marrow mesenchymal stem cells. Mol Cell Biochem 362: 25-33, 2012.

5. Jung CH, Cho I, Ahn J, Jeon TI and Ha TY: Quercetin reduces high-fat diet-induced fat accumulation in the liver by regulating lipid metabolism genes. Phytother Res 27: 139-143, 2013.

6. Zhang H, Zhang M, Yu L, Zhao Y, He N and Yang X: Antitumor activities of quercetin and quercetin-5',8-disulfonate in human colon and breast cancer cell lines. Food Chem Toxicol 50: 1589-1599, 2012

7. Wong MY and Chiu GN: Liposome formulation of co-encapsulated vincristine and quercetin enhanced antitumor activity in a trastuzumab-insensitive breast tumor xenograft model. Nanomedicine 7: 834-840, 2011.
8. Niklas J, Nonnenmacher Y, Rose T, Sandig V and Heinzle E: Quercetin treatment changes fluxes in the primary metabolism and increases culture longevity and recombinant $\alpha 1$-antitrypsin production in human AGE1.HN cells. Appl Microbiol Biotechnol 94: 57-67, 2012.

9. Liang W, Luo Z, Ge S, Li M, Du J, Yang M, Yan M, Ye Z and Luo Z: Oral administration of quercetin inhibits bone loss in rat model of diabetic osteopenia. Eur J Pharmacol 670: 317-324, 2011.

10. Yamaguchi $M$ and Weitzmann MN: Quercetin, a potent suppressor of NF- $x \mathrm{~B}$ and Smad activation in osteoblasts. Int J Mol Med 28: 521-525, 2011.

11. Sharan K, Mishra JS, Swarnkar G, Siddiqui JA, Khan K, Kumari R, Rawat P, Maurya R, Sanyal S and Chattopadhyay N: A novel quercetin analogue from a medicinal plant promotes peak bone mass achievement and bone healing after injury and exerts an anabolic effect on osteoporotic bone: the role of aryl hydrocarbon receptor as a mediator of osteogenic action. J Bone Miner Res 26: 2096-2111, 2011.

12. Liang SZ, Wang GX, Fan LK, Wu GY, Jin Y and Zhu GX: Proliferation and differentiation of bone marrow mesenchymal stem cells in process of bone loss in ovariectomized rats. Zhongguo Bing Li Sheng Li Za Zhi 28: 398-403, 2012 (In Chinese)

13. Yamaguchi A, Komori $\mathrm{T}$ and Suda $\mathrm{T}$ : Regulation of osteoblast differentiation mediated by bone morphogenetic peoteins, hedgehogs, and Cbfa1. Endocr Rev 21: 393-411, 2000.

14. Palcy S and Goltzman D: Protein kinase signalling pathways involved in the up- regulation of the rat $\alpha 1$ (I) collagen gene by transforming growth factor $\beta 1$ and bone morphogenetic protein 2 in osteoblastic cells. Biochem J 343: 21-27, 1999.

15. Park YJ, Lee JM, Shin SY and Kim YH: Constitutively active Ras negatively regulates Erk MAP kinase through induction of MAP kinase phosphatase 3 (MKP3) in NIH3T3 cells. BMB Rep 47: 685-690, 2014.

16. Sheng XY: Research progress of ERK5 signaling pathway in the MAPK family. Yi Xue Zongshu 18: 3145-3147, 2012 (In Chinese).

17. Zhou H, Yang X, Wang N, Zhang Y and Cai G: Tigogenin inhibits adipocytic differentiation and induces osteoblastic differentiation in mouse bone marrow stromal cells. Mol Cell Endocrinol 270: 17-22, 2007.

18. Canalis E: Osteogenic growth factors. In: Primer on the Metabolic Bone Diseases and Disorders of Mineral Metabolism. Favus MJ (ed). 5th edition. American Society for Bone and Mineral Research, Washington DC, pp28-31, 2003.

19. Tian H, Bi X, Li CS, Zhao KW, Brochmann EJ, Montgomery SR, Aghdasi B, Chen D, Daubs MD, Wang JC and Murray SS: Secreted phosphoprotein $24 \mathrm{kD}$ (Spp24) and Spp14 affect TGF- $\beta$ induced bone formation differently. PLoS One 8: e72645, 2013.

20. Palcy S, Bolivar I and Goltzman D: Role of activator protein 1 transcriptional activity in the regulation of gene expression by transforming growth factor beta1 and bone morphogenetic protein 2 in ROS 17/2.8 osteoblast-like cells. J Bone Miner Res 15: 2352-2361, 2000.

21. Guicheux J, Lemonnier J, Ghayor C, Suzuki A, Palmer G and Caverzasio J: Activation of p38 mitogen-activated protein kinase and c-Jun-NH2-terminal kinase by BMP-2 and their implication in the stimulation of osteoblastic cell differentiation. J Bone Miner Res 18: 2060-2068, 2003.

22. Lee J, Roh KB, Kim SC, Lee J and Park D: Soy peptide-induced stem cell proliferation: involvement of ERK and TGF- $\beta 1$. J Nutr Biochem 23: 1341-1351, 2012.

23. Peng S, Zhou G, Luk KD, Cheung KM, Li Z, Lam WM, Zhou Z and $\mathrm{Lu} \mathrm{WW}$ : Strontium promotes osteogenic differentiation of mesenchymal stem cells through the Ras/MAPK signaling pathway. Cell Physiol Biochem 23: 165-174, 2009.

24. Takeda S, Bonnamy JP, Owen MJ, Ducy P and Karsenty G: Continuous expression of Cbfa1 in nonhypertrophic chondrocytes uncovers its ability to induce hypertrophic chondrocyte differentiation and partially rescues Cbfa1-deficient mice. Genes Dev 15: 467-481, 2001.

25. Wang XY, Wang YH, Zhang DG, Li JJ, Cui L, Shi B, Liu WG and Wang XZ: Study on the regulation of Cbf $\alpha 1$ to definitive differentiation of bone marrow mesenchymal stem cells. Zhongguo Linchuang Kangfu 7: 3164-3165, 2003. 